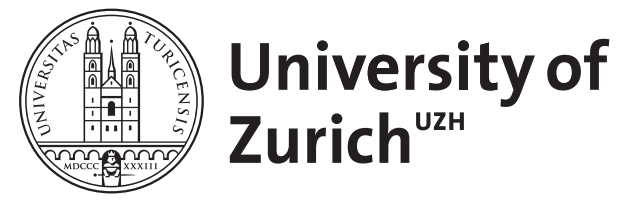

\title{
Local times for functions with finite variation: two versions of Stieltjes change-of-variables formula
}

\author{
Bertoin, Jean ; Yor, Marc
}

DOI: https://doi.org/10.1112/blms/bdu014

Posted at the Zurich Open Repository and Archive, University of Zurich ZORA URL: https://doi.org/10.5167/uzh-98953

Journal Article

Published Version

Originally published at:

Bertoin, Jean; Yor, Marc (2014). Local times for functions with finite variation: two versions of Stieltjes change-of-variables formula. Bulletin of the London Mathematical Society, 46(3):553-560.

DOI: https://doi.org/10.1112/blms/bdu014 


\title{
Local times for functions with finite variation: two versions of Stieltjes change-of-variables formula
}

\author{
Jean Bertoin and Marc Yor
}

\begin{abstract}
We introduce two natural notions for the occupation measure of a function $V$ with finite variation. The first yields a signed measure, and the second a positive measure. By comparing two versions of the change-of-variables formula, we show that both measures are absolutely continuous with respect to the Lebesgue measure. Occupation densities can be thought of as local times of $V$, and are described by a Meyer-Tanaka like formula. As an example of applications, we derive an extension of Banach's indicatrix theorem.
\end{abstract}

\section{Introduction}

Although the setting of the present work is entirely deterministic and does not involve Probability Theory at all, its motivation comes from an important chapter of Stochastic Calculus (see, for instance, Protter [8, Section IV.7]). Specifically, let $\left(X_{t}\right)_{t \geqslant 0}$ be a real-valued semimartingale, that is, $X=M+V$ where $M$ is a local martingale and $V$ a process with finite variation, and the paths of $X$ are right-continuous and possess left-limits (càdlàg) almost surely (a.s.). In turn, the local martingale can be expressed as the sum $M=M^{c}+M^{d}$ of a continuous local martingale $M^{c}$ and a purely discontinuous local martingale $M^{d}$. The occupation measure of $X$ on a time interval $[0, t]$ is defined as

$$
\int_{0}^{t} \mathbf{1}_{A}\left(X_{s}\right) d\left\langle M^{c}\right\rangle_{s}, \quad A \in \mathcal{B}(\mathbb{R}),
$$

where $\left\langle M^{c}\right\rangle$ denotes the quadratic variation of $M^{c}$. A fundamental result in this area is that this occupation measure is a.s. absolutely continuous with respect to the Lebesgue measure, that is, the following occupation density formula holds:

$$
\int_{0}^{t} \mathbf{1}_{A}\left(X_{s}\right) d\left\langle M^{c}\right\rangle_{s}=\int_{A} L_{t}^{x} d x
$$

The occupation densities $\left\{L_{t}^{x}: x \in \mathbb{R}\right.$ and $\left.t \geqslant 0\right\}$ are known as the local times of $X$ and are described by the Meyer-Tanaka formula

$$
\begin{aligned}
& \left(X_{t}-x\right)^{+}-\left(X_{0}-x\right)^{+} \\
& \quad=\int_{0}^{t} \mathbf{1}_{\left\{X_{s-}>x\right\}} d X_{s}+\frac{1}{2} L_{t}^{x}+\sum_{0<s \leqslant t}\left(\mathbf{1}_{\left\{X_{s-} \leqslant x\right\}}\left(X_{s}-x\right)^{+}+\mathbf{1}_{\left\{X_{s-}>x\right\}}\left(X_{s}-x\right)^{-}\right) .
\end{aligned}
$$

The purpose of this work is to point out that the above results for semimartingales have natural analogs in the deterministic world of functions with finite variation. The rest of this note is organized as follows. Our main result is stated in Section 2 and proved in Section 4. Section 3 is devoted to two versions of the Stieltjes change-of-variables formula which lie at the heart of the analysis. Finally, some applications, in particular related to Banach's Indicatrix Theorem, are discussed in Section 5.

Received 19 June 2013; revised 12 November 2013; published online 30 March 2014.

2010 Mathematics Subject Classification 26A45, 60J55 (primary). 


\section{Local times for functions with finite variation}

We consider a càdlàg function $V:[0, \infty) \rightarrow \mathbb{R}$ with finite variation. There is the canonical decomposition of $V$ as the sum of its continuous and discontinuous components,

$$
V=V^{c}+V^{d}
$$

where

$$
V^{d}(t)=\sum_{0<s \leqslant t} \Delta V(s)
$$

and the series is absolutely convergent. Plainly, the continuous part $V^{c}$ has also finite variation; we write $V^{c}(d t)$ for the corresponding signed Stieltjes measure and $\left|V^{c}(d t)\right|$ for its total variation measure.

We then introduce for every $t>0$ a signed measure $\theta_{t}(d x)$ and a positive measure $\vartheta_{t}(d x)$, which are defined, respectively, by

$$
\theta_{t}(A)=\int_{0}^{t} \mathbf{1}_{A}(V(s)) V^{c}(d s)
$$

and

$$
\vartheta_{t}(A)=\int_{0}^{t} \mathbf{1}_{A}(V(s))\left|V^{c}(d s)\right|
$$

where $A \subseteq \mathbb{R}$ stands for a generic Borel set. We interpret $\theta_{t}(d x)$ and $\vartheta_{t}(d x)$, respectively, as the signed and the absolute occupation measure of $V$ on the time interval $[0, t]$.

Our goal is to show that the occupation measures $\theta_{t}(d x)$ and $\vartheta_{t}(d x)$ are both absolutely continuous with respect to the Lebesgue measure and to describe explicitly their densities. In this direction, we introduce the following definitions and notation.

For every $x \in \mathbb{R}$ and $t>0$, we first set

$$
n^{x}(t)=\operatorname{Card}\{0 \leqslant s \leqslant t: V(s)=x\},
$$

for the number of passages at level $x$ before time $t$. Next, we say that $V$ increases continuously through the level $x$ at time $t$ and then write $t \in \mathcal{I}(x)$ if:

(i) $V(t)=x$ and $V$ is continuous at time $t$;

(ii) $V(s)-V(t)$ has the same sign as $s-t$ for all $s$ in some neighborhood of $t$.

Similarly, we say that $V$ decreases continuously through the level $x$ at time $t$ and then write $t \in \mathcal{D}(x)$ if $-V$ increases through the level $-x$ at time $t$. We then define

$$
\ell^{x}(t)=\operatorname{Card}\{(0, t] \cap \mathcal{I}(x)\}-\operatorname{Card}\{(0, t] \cap \mathcal{D}(x)\},
$$

whenever the two quantities in the difference in the right-hand side are finite. For each such $x, t \rightarrow \ell^{x}(t)$ is a right-continuous function with integer values, which can only jump at times when $V$ reaches $x$ continuously. In that case, we also set

$$
\lambda^{x}(t)=\operatorname{Card}\{(0, t] \cap \mathcal{I}(x)\}+\operatorname{Card}\{(0, t] \cap \mathcal{D}(x)\},
$$

so that the point measure $\lambda^{x}(d t)$ coincides with $\left|\ell^{x}(d t)\right|$, the total variation measure of $\ell^{x}(d t)$.

Our main result identifies $\left(\ell^{x}(t): x \in \mathbb{R}\right.$ and $\left.t \geqslant 0\right)$ and $\left(n^{x}(t): x \in \mathbb{R}\right.$ and $\left.t \geqslant 0\right)$, respectively, as the signed and absolute local times of $V$.

TheOREM 1. For every $t>0$, there are the identities

$$
\theta_{t}(d x)=\ell^{x}(t) d x \quad \text { and } \quad \vartheta_{t}(d x)=n^{x}(t) d x=\lambda^{x}(t) d x .
$$


Theorem 1 is an immediate consequence of the chain rule when $V$ is piecewise of class $\mathcal{C}^{1}$ (with possible jumps); however, the general case requires a more delicate analysis. It is of course implicit in the statement that $\ell^{x}(t)$ and $n^{x}(t)$ are well defined for almost all $x \in \mathbb{R}$ and yield measurable functions in $L^{1}(d x)$, which is not a priori obvious.

It is interesting to point out that essentially the same result was established by Fitzsimmons and Port [6] in the special case when $V$ is a Lévy process with finite variation and nonzero drift (then, when for instance the drift is positive, only increasing passages through a level may occur). In the same direction, Theorem 1 applies much more generally to sample paths of a large class of (semi) martingales with finite variation, which arise for instance by predictable compensation of a pure jump process with finite variation. Note that when $M$ is a local martingale with finite variation, then its continuous component in the sense of Martingale Theory is always degenerate (that is, identically zero), whereas its continuous component in the sense of functions with finite variation may be non-trivial. In that case, the occupation measure defined by (1) is degenerate, and then definitions (3) and (4) may be sounder.

We further stress that for semimartingales with non-degenerate continuous martingale component $M^{c} \not \equiv 0$, the local time $t \rightarrow L_{t}^{x}$ defines an increasing process that is always continuous, whereas for functions with finite variation, $t \rightarrow \ell^{x}(t)$ and $t \rightarrow n^{x}(t)$ are integer-valued step-functions.

\section{Two versions of the change-of-variables formula}

Our strategy for proving Theorem 1 is to compare two change-of-variables formulas. The first version is standard and its proof is therefore omitted.

Proposition 1. Let $f: \mathbb{R} \rightarrow \mathbb{R}$ be a function of class $\mathcal{C}^{1}$. Then for every $t>0$, there is the identity

$$
f(V(t))-f(V(0))=\int_{0}^{t} f^{\prime}(V(s)) V^{c}(d s)+\sum_{0<s \leqslant t}(f(V(s))-f(V(s-))) .
$$

REMARK 1. Itô's formula for càdlàg processes with finite variation reads in the setting of Proposition 1

$$
f(V(t))-f(V(0))=\int_{[0, t]} f^{\prime}(V(s-)) V(d s)+\sum_{0<s \leqslant t}\left(f(V(s))-f(V(s-))-f^{\prime}(V(s-)) \Delta V(s)\right) .
$$

See, for instance, Dellacherie and Meyer [4, pp. 168-171], and in particular the formula (92.1) there. Of course, one then recovers Proposition 1 by observing that

$$
\int_{[0, t]} f^{\prime}(V(s-)) V^{d}(d s)=\sum_{0<s \leqslant t} f^{\prime}(V(s-)) \Delta V(s),
$$

and that

$$
\int_{[0, t]} f^{\prime}(V(s-)) V^{c}(d s)=\int_{[0, t]} f^{\prime}(V(s)) V^{c}(d s),
$$

since the set of discontinuities of $V$ is at most countable and the continuous Stieltjes measure $V^{c}(d s)$ assigns no mass to countable sets. 
In order to state a second version of the change-of-variables formula, we need first to introduce some further notions. We say that $x \in \mathbb{R}$ is a simple level for $V$ if the set

$$
\{t>0: V(t-)<x<V(t) \text { or } V(t)<x<V(t-) \text { or } V(t)=x\}
$$

is discrete and if there is no jump of $V$ that starts or ends at $x$, that is,

$$
\{t>0: \Delta V(t) \neq 0 \text { and either } x=V(t) \text { or } x=V(t-)\}=\emptyset .
$$

Otherwise, we say that $x$ is a complex level for $V$.

Lemma 1. The set of complex levels for $V$ has zero Lebesgue measure.

Proof. The set of jump times of $V$ is at most countable. Thus, so is

$$
\{x \in \mathbb{R}: x=V(t) \text { or } x=V(t-) \text { for some } t>0 \text { with } \Delta V(t) \neq 0\},
$$

and a fortiori this set has zero Lebesgue measure.

Consider a sequence of partitions $\tau_{n}$ of $[0, \infty)$ with mesh tending to 0 and such that $\tau_{n+1}$ is thinner than $\tau_{n}$. We write $0=t_{0}^{n}<t_{1}^{n}<\cdots$ for the elements of $\tau_{n}$. We set for every $n, i \in \mathbb{N}$

$$
J_{i}^{n}=\left[\inf _{t_{i}^{n} \leqslant s \leqslant t_{i+1}^{n}} V(s), \sup _{t_{i}^{n} \leqslant s \leqslant t_{i+1}^{n}} V(s)\right],
$$

and write as usual $\left|J_{i}^{n}\right|$ for the length of this interval. If we write $W(t)$ for the total variation of $V$ on $[0, t]$, then for every $t \in \tau_{n}$, there is the upper bound

$$
\sum_{i: t_{i}^{n}<t}\left|J_{i}^{n}\right| \leqslant W(t)
$$

Now introduce the number of passages at or across $x$ before time $t$,

$$
N_{x}(t)=\operatorname{Card}\{0<s \leqslant t: V(s-) \leqslant x \leqslant V(s) \text { or } V(s) \leqslant x \leqslant V(s-)\},
$$

and observe that

$$
N_{x}(t)=\lim _{n \rightarrow \infty} \uparrow \sum_{i: t_{i}^{n}<t} \mathbf{1}_{J_{i}^{n}}(x) .
$$

It follows from monotone convergence and Fubini-Tonelli Theorem that

$$
\int_{\mathbb{R}} N_{x}(t) d x \leqslant W(t)<\infty .
$$

A fortiori $N_{x}(t)<\infty$ for almost every $x$, which entails that the set

$$
\{t>0: V(t-) \leqslant x \leqslant V(t) \text { or } V(t) \leqslant x \leqslant V(t-)\}
$$

is discrete for almost every $x$.

We stress that for every simple level $x$, we can enumerate the increasing and the decreasing passage times of $V$ through $x$, and $\ell^{x}(t), \lambda^{x}(t)$ and $n^{x}(t)$ are all well defined and finite. We are now able to state the second version of the change-of-variables formula.

Proposition 2. Let $f: \mathbb{R} \rightarrow \mathbb{R}$ be a function of class $\mathcal{C}^{1}$. Then for every $t>0$, there is the identity

$$
f(V(t))-f(V(0))=\int_{\mathbb{R}} f^{\prime}(x) \ell^{x}(t) d x+\sum_{0<s \leqslant t}(f(V(s))-f(V(s-))) .
$$


Remark 2. Our recent note [3, Theorem 2.1(i)] contains a similar result when $V$ is an increasing process and the function $f$ is merely assumed to be non-decreasing. Of course, when $V$ is non-decreasing, the number of increasing passages through any level is at most 1 and there are no decreasing passages, so $\ell^{x}(t)=1$ or 0 , depending essentially on whether $V$ hits the level $x$ before time $t$ or not.

Proof. Consider a simple level $x$. We further suppose that $x \neq V(t)$ and $x \neq V(0)$. For any such $x$, one can define the consecutive crossings of the level $x$ by the graph of $V$. Each crossing can be made either upwards or downwards, and occurs either continuously or by a jump. More precisely, an upward crossing of $x$ corresponds either to an increase through the level $x$, or to a positive jump starting strictly below $x$ and finishing strictly above $x$; and there is an analogous alternative for downwards crossings. Plainly upwards and downwards crossings alternate, and a short moment of reflexion shows that there is the identity

$$
\mathbf{1}_{[x, \infty)} V(t)=\mathbf{1}_{[x, \infty)} V(0)+\ell^{x}(t)+\sum_{0<s \leqslant t}\left(\mathbf{1}_{[x, \infty)} V(s)-\mathbf{1}_{[x, \infty)} V(s-)\right),
$$

where the series in the right-hand side accounts for the difference between the number of positive jumps and the number of negative jumps across the level $x$.

It is convenient to write $\nu$ for the point measure on $[0, \infty)$ that has an atom at each jump time of $V$, viz.

$$
\nu(d t)=\sum_{s \geqslant 0} \mathbf{1}_{\{\Delta V(s) \neq 0\}} \delta_{s}(d t) .
$$

We stress that $\nu$ is sigma-finite (because the number of jump times of $V$ is at most countable), and rewrite

$$
\sum_{0<s \leqslant t}\left(\mathbf{1}_{[x, \infty)} V(s)-\mathbf{1}_{[x, \infty)} V(s-)\right)=\int_{[0, t]}\left(\mathbf{1}_{[x, \infty)} V(s)-\mathbf{1}_{[x, \infty)} V(s-)\right) \nu(d s) .
$$

We also observe that, in the notation of the proof of Lemma 1, there is the inequality

$$
\int_{[0, t]}\left|\mathbf{1}_{[x, \infty)} V(s)-\mathbf{1}_{[x, \infty)} V(s-)\right| \nu(d s) \leqslant N_{x}(t),
$$

and recall from (5) that the function $x \rightarrow N_{x}(t)$ is in $L^{1}(d x)$. Because the map

$$
(s, x) \longrightarrow\left(\mathbf{1}_{[x, \infty)} V(s)-\mathbf{1}_{[x, \infty)} V(s-)\right)
$$

is measurable, it follows from Fubini Theorem that the map

$$
x \longrightarrow \int_{[0, t]}\left(\mathbf{1}_{[x, \infty)} V(s)-\mathbf{1}_{[x, \infty)} V(s-)\right) \nu(d s)
$$

is also measurable. In particular, we conclude from (6) that $x \rightarrow \ell^{x}(t)$ is measurable.

We now rewrite the identity (6) in the form

$$
\mathbf{1}_{[x, \infty)} V(t)=\mathbf{1}_{[x, \infty)} V(0)+\ell^{x}(t)+\int_{[0, t]}\left(\mathbf{1}_{[x, \infty)} V(s)-\mathbf{1}_{[x, \infty)} V(s-)\right) \nu(d s),
$$

multiply both sides by $f^{\prime}(x)$ and integrate with respect to the Lebesgue measure $d x$. An application of Fubini Theorem (which is legitimate thanks to the observations above) yields

$$
f(V(t))=f(V(0))+\int_{\mathbb{R}} f^{\prime}(x) \ell^{x}(t) d x+\int_{[0, t]}(f(V(s))-f(V(s-))) \nu(d s),
$$

which is the change-of-variables formula of the statement. 


\section{Proof of Theorem 1}

We now turn our attention to the proof of our main result, which will also require the following elementary observation.

Lemma 2. Let $\mu$ be a signed measure on some measurable space, and $\varphi$ be a measurable function with values in $\{-1,1\}$ such that $\varphi \mu$ is a positive measure. Then $\varphi \mu$ coincides with the total variation measure $|\mu|$.

Proof. Indeed, $\varphi \mu=|\varphi \mu|$ since $\varphi \mu$ is a positive measure, and on the other hand, we have also $|\varphi \mu|=|\varphi||\mu|=|\mu|$ since $|\varphi| \equiv 1$.

We now have all the ingredients needed for establishing Theorem 1.

Proof of Theorem 1. First, comparing the two change-of-variables formulas in Propositions 1 and 2, we get that for any continuous function $g: \mathbb{R} \rightarrow \mathbb{R}$ (think of $g=f^{\prime}$ as the derivative of a $\mathcal{C}^{1}$ function), there is the identity

$$
\int_{0}^{t} g(V(s)) V^{c}(d s)=\int_{\mathbb{R}} g(x) \theta_{t}(d x)=\int_{\mathbb{R}} g(x) \ell^{x}(t) d x .
$$

Thus, $\theta_{t}(d x)=\ell^{x}(t) d x$.

Next, we introduce the signed measure $\mu(d s, d x)$ on $[0, t] \times \mathbb{R}$ which is defined by

$$
\mu(A)=\int_{0}^{t} \mathbf{1}_{A}(s, V(s)) V^{c}(d s), \quad A \in \mathcal{B}([0, t] \times \mathbb{R}) .
$$

The (signed) occupation density formula above yields that for every $f: \mathbb{R} \rightarrow \mathbb{R}$ and $g:[0, t] \rightarrow \mathbb{R}$ measurable and bounded, there is the identity

$$
\int_{[0, t] \times \mathbb{R}} f(x) g(s) \mu(d s, d x)=\int_{0}^{t} f(V(s)) g(s) V^{c}(d s)=\int_{\mathbb{R}}\left(\int_{[0, t]} g(s) \ell^{x}(d s)\right) f(x) d x .
$$

More precisely, when $g$ is a step function, this identity follows from the occupation density formula by linearity, and the general case is then derived through a version of the monotone class theorem (cf. Neveu $[\mathbf{7}]$ ).

Then consider a measurable function $\varphi:[0, \infty) \rightarrow\{1,-1\}$ such that $V^{c}(d t)=\varphi(t)\left|V^{c}(d t)\right|$. We have

$$
\int_{[0, t] \times \mathbb{R}} f(x) g(s) \varphi(s) \mu(d s, d x)=\int_{0}^{t} f(V(s)) g(s)\left|V^{c}(d s)\right|,
$$

and the right-hand side is non-negative whenever $f, g \geqslant 0$. Again by a version of the monotone class theorem, this shows that $\varphi(s) \mu(d s, d x)$ is a positive measure, and more precisely, since $|\varphi|=1$, Lemma 2 shows that $\varphi(s) \mu(d s, d x)=|\mu(d s, d x)|$ is the total variation measure of $\mu(d s, d x)$. Now we write

$$
\int_{0}^{t} f(V(s)) g(s)\left|V^{c}(d s)\right|=\int_{\mathbb{R}}\left(\int_{[0, t]} g(s) \varphi(s) \ell^{x}(d s)\right) f(x) d x .
$$

Plainly, whenever $g \geqslant 0$, we must have $\int_{[0, t]} g(s) \varphi(s) \ell^{x}(d s) \geqslant 0$ for almost all $x \in \mathbb{R}$, and it follows easily that $\varphi(s) \ell^{x}(d s)$ is a positive measure for almost all $x \in \mathbb{R}$. Again because $|\varphi|=1$, this entails that $\varphi(s) \ell^{x}(d s)$ coincides with the total variation measure $\left|\ell^{x}(d t)\right|$, that is,

$$
\varphi(s) \ell^{x}(d s)=\left|\ell^{x}(d s)\right|=\lambda^{x}(d s) .
$$


Putting the pieces together, we have shown that

$$
\int_{0}^{t} f(V(s)) g(s)\left|V^{c}(d s)\right|=\int_{\mathbb{R}}\left(\int_{[0, t]} g(s) \lambda^{x}(d s)\right) f(x) d x,
$$

which for $g \equiv 1$ simply reads $\vartheta_{t}(d x)=\lambda^{x}(t) d x$.

To complete the proof, we observe that the identity

$$
\lambda^{x}(t)=n^{x}(t) \text { for all } t \geqslant 0
$$

holds whenever the level $x$ is simple and $x$ is not the value of a local extremum of $V$. Since the set of local extrema is at most countable, we deduce from Lemma 1 that (7) holds for almost every $x$, and we conclude that $\vartheta_{t}(d x)=n^{x}(t) d x$.

We now conclude this section by stressing that the elementary identity (6) for the signed local time $\ell^{x}(t)$ at a simple level $x$ should be viewed as the analog of the Meyer-Tanaka formula (2) in the semimartingale setting. We also point at the alternative formula

$$
\mathbf{1}_{(-\infty, x)} V(t)=\mathbf{1}_{(-\infty, x)} V(0)-\ell^{x}(t)+\sum_{0<s \leqslant t}\left(\mathbf{1}_{(-\infty, x)} V(s)-\mathbf{1}_{(-\infty, x)} V(s-)\right) .
$$

For the absolute local time $n^{x}(t)$, one sees similarly that for every simple level $x$ that is not a local extrema of $V$, there is the identity

$$
H^{x}(t)=n^{x}(t)+\sum_{0<s \leqslant t}\left|\mathbf{1}_{[x, \infty)} V(s)-\mathbf{1}_{[x, \infty)} V(s-)\right|,
$$

where $H^{x}(t)$ denotes the total variation on the time interval $[0, t]$ of the step function $\mathbf{1}_{[x, \infty)} \circ V$.

\section{Some applications}

We point at the following immediate consequence of Theorem 1.

Corollary 1. The total variation of the continuous component $V^{c}$ of $V$ on the interval $[0, t]$ can be expressed as

$$
\int_{-\infty}^{\infty} n^{x}(t) d x
$$

Equivalently, the total variation of $V$ on $[0, t]$ is given by

$$
\int_{-\infty}^{\infty} n^{x}(t) d x+\sum_{0<s \leqslant t}|\Delta V(s)|
$$

When $V$ is continuous, this is known as Banach's Indicatrix Theorem; see [1]. In the same vein, we also point out the following identity for the continuous part of $V$ :

$$
V^{c}(t)-V^{c}(0)=\int_{-\infty}^{\infty} \ell^{x}(t) d x
$$

In another direction, we point out that if $V$ is not purely discontinuous, that is, $V^{c} \not \equiv 0$, then the set of levels through which $V$ increases or decreases continuously has a positive Lebesgue measure. This contrasts sharply with the case of a typical Brownian path, or also, say, a sample path of a symmetric stable Lévy process with index $\alpha \in(1,2)$, as then local times exist, but there is no level through which the sample path increases or decreases (cf. Dvoretsky et al. [5] and [2]). Of course, in those examples, sample paths have infinite variation. 
In the same vein, recall from Section 2 that for every $x \in \mathbb{R}$, we write $\mathcal{I}(x)$ (respectively, $\mathcal{D}(x)$ ) for the set of times at which $V$ increases (respectively, decreases) continuously across the level $x$.

Corollary 2. Suppose that the continuous component $V^{c}$ of $V$ is non-decreasing. Then for almost all levels $x$, there are the identities

$$
\mathcal{I}(x)=\{t \geqslant 0: V(t)=x\} \quad \text { and } \quad \mathcal{D}(x)=\emptyset .
$$

Proof. When $V^{c}$ is non-decreasing, $V^{c}(d t)=\left|V^{c}(d t)\right|$ and therefore $\theta_{t}(d x)=\vartheta_{t}(d x)$. Theorem 1 entails that $\ell^{x}(t)=\lambda^{x}(t)=n^{x}(t)$ for almost all $x \in \mathbb{R}$, and our claim follows easily.

Acknowledgements. We would like to thank Pat Fitzsimmons and Youssef Ouknine for pointing at the connection of Theorem 1 with Banach's Indicatrix Theorem.

\section{References}

1. S. BANACH, 'Sur les lignes rectifiables et les surfaces dont l'aire est finie', Fund. Math. 7 (1925) 225-236.

2. J. Bertoin, 'Increase of stable processes', J. Theoret. Prob. 7 (1994) 551-563.

3. J. Bertoin and M. Yor, 'Pure jump increasing processes and the change of variables formula', Electron. Comm. Probab. 18 (2013) 1-7.

4. C. Dellacherie and P. A. Meyer, 'Probabilités et potentiel. Chapitres V à VIII', Actualités Scientifiques et Industrielles [Current Scientific and Industrial Topics] 1385 (Hermann, Paris, 1980).

5. A. Dvoretzky, P. Erdös and S. Kakutani, 'Nonincrease everywhere of the Brownian motion process', Proc. 4th Berkeley Symp. Math. Stat. Prob. II (University of California Press, Berkeley, California, 1961) 103-116.

6. P. J. Fitzsimmons and S. C. Port, 'Local times, occupation times, and the Lebesgue measure of the range of a Lévy process', Seminar on Stochastic Processes, 1989 (San Diego, CA, 1989), Progress in Probability 18 (Birkhäuser Boston, Boston, 1990) 59-73.

7. J. Neveu, Mathematical foundations of the calculus of probability (Holden-Day, Inc., San Francisco, CA, 1965).

8. Ph. Protter, Stochastic integration and differential equations, Applications of Mathematics 21 (Springer, New York, 2004).

Jean Bertoin

Institut für Mathematik

Universität Zürich

Winterthurerstrasse 190

CH-8057 Zürich

Switzerland

jean.bertoin@math.uzh.ch

\author{
Marc Yor \\ Laboratoire de Probabilités et Modèles \\ Aléatoires \\ Institut Universitaire de France \\ UPMC \\ 4 Place Jussieu \\ 75252 Paris cedex 05 \\ France
}

deaproba@proba.jussieu.fr 